\title{
Bioinformation
}

\section{Towards reconstructing a metabolic tree of life}

\author{
Marina Marcet-Houben ${ }^{1}$, Pere Puigbò ${ }^{1}$, Antoni Romeu ${ }^{1}$ and Santiago Garcia-Vallve ${ }^{1, *}$ \\ ${ }^{1}$ Evolutionary Genomics Group, Biochemistry and Biotechnology Department, Rovira i Virgili University, Campus Sescelades, c/ Marcel li Domingo \\ s/n, 43007 TARRAGONA, Spain; Santiago Garcia-Vallve* - E-mail: santi.garcia-vallve@urv.cat; * Corresponding author
}

received November 17, 2007; accepted November 23, 2007; published online December 11, 2007

\begin{abstract}
:
Using information from several metabolic databases, we have built our own metabolic database containing 434 pathways and 1157 different enzymes. We have used this information to construct a dendrogram that demonstrates the metabolic similarities between 282 species. The resulting species distribution and the clusters defined in the tree show a certain taxonomic congruence, especially in recent relationships between species. This dendrogram is another representation of the tree of life, based on metabolism that may complement the trees constructed by other methods. For example, the metabolic dissimilarity we demonstrate between Symbiobacterium thermophilum (previously defined as Actinobacteria) and the other Actinobacteria species, and the metabolic similarity between $S$. thermophilum and Clostridia, combined with other evidence, suggest that $S$. thermophilum may be re-classified as Firmicutes, Clostridia.
\end{abstract}

Keywords: metablic pathways; enzymes; dendogram; taxonomy; species

\section{Background:}

For many years phylogenetic trees have been used to study the evolution of organisms. Since Charles Darwin first described the evolution of species as a tree, scientist have attempted to create a tree that could represent a hierarchical classification of all known species based on their evolution and at the same time provide information about extinct species and the common ancestry shared by known species. When sequencing technologies were developed, the use of taxonomic marker molecules such as the small subunit ribosomal RNA seemed sufficient to draw consistent phylogenetic trees. Studies using genes or protein sequences led to a classification of microorganisms and recognised the Archaea as the third domain of life. [1]

When whole genome sequences of prokaryote organisms became available, everyone hoped that this extended information would help them to build more accurate phylogenies but it was then discovered that different genes produced different trees. It was at this point that doubts were raised as to whether a tree structure was the best representation of evolution. [2] Simultaneously, the discovery that horizontal gene transfer events (HGT) between species was more common than previously suspected $[3,4]$ put a strain on the search for the "true tree". [5] After all, the gene used in a phylogenetic study may very well have been acquired from an organism that was in no way a direct ancestor. [6] In view of the above, some scientists have started to consider that evolution is perhaps better represented by a network than by a tree. [7] Studies have also begun into new ways of creating a universal tree of life. If taking a single gene had become insufficient for consistent tree representation, now that hundreds of whole genomic sequences are available, new phylogenomic methods are being developed. [8] As it is difficult to align the sequences of two genomes, several methods that use traditional sequence alignment tools have been developed to construct genome trees. $[\mathbf{8}, \mathbf{9 , 1 0 ]}$ These methods involve concatenating the homologous sequences from different gene families to construct a single tree $[\mathbf{9}, \mathbf{1 0}, \mathbf{1 1}]$ or comparing different trees to create a supertree. [12] Another way to describe the relationships between genomes is to use their gene repertoire. [13] New methods based on gene order or gene content have therefore been developed. [10] The main problem with these methods is the imbalance in the number of genes between small and large genomes. Two large genomes that are not phylogenetically closely related can have more common genes than a large and a small genome that are closely related. Measures to prevent this must be taken so that the phylogenetic tree does not become biased. [10]

Genome trees seem to reveal a phylogenetic signal that supports the three-domain evolutionary scenario and the relationships between some clades of Bacteria. However, deep-level prokaryotic relationships are difficult to infer. [12] We have developed a new method for constructing a genome tree based on the metabolic pathways present in each species. The main structure of the metabolic pathways seems to be largely unaffected by HGT. [14] This enables us to use them as templates for comparing genomes. Using the orthologous groupings of enzymes found in the KEGG database, we have related genomes and metabolic pathways and created a tree-like representation of a fairly large group of organisms based on their metabolism.

\section{Methodology:}

Our aim was to create a dendrogram of different eukaryotic and prokaryotic species based on metabolic data. Here we detail the characteristics of the process used:

\section{Database creation}

Starting from the metabolic maps available in the KEGG: Kyoto Encyclopedia of Genes and Genomes [15] (http://www.genome.ad.jp/kegg/) and the MetaCyc [16] (http://www.metacyc.org) databases, we defined a representative group of pathways and introduced into our database the enzymes that catalyse each of the reactions that form every pathway by their KO number as defined in KEGG. Since a same pathway can follow slightly different routes in different organisms, we 


\section{Bioinformation}

www.bioinformation.net

added different variants to some of the pathways. For example, we introduced five variants of the glycolysis pathway. At the end, our database contained 434 pathways and 1157 enzymes with different $\mathrm{KO}$ numbers.

\section{Percentage matrix}

The next step was to relate the data found in our database to a group of organisms. We used the complete genomes found in the KEGG database. For each organism, we created a list of enzymes codified in the genome, listed by their K number. Since the KEGG database is still growing and new genomes are being introduced, some of them still did not have all their KEGG numbers assigned. So, we compared the number of proteins with an assigned KEGG number to the total number of proteins coded in each genome. Those organisms in which the assigned number of proteins in the KEGG database was less than 20 percent were excluded from the list of organisms used to build the dendrogram. Finally we took 282 organisms which are listed in supplementary Table 1 with their abbreviation. Using information from the metabolic database we had previously created, we searched in each genome for the enzymes that completed each pathway. To do so, we made a PERL script that calculated the percentages of enzymes that appeared in a pathway for each organism. The results were presented in a matrix whose rows were the pathways, whose columns were the organisms analysed and in which each element represented the percentage of enzymes of a pathway that one organism contains.

\section{Dendrogram construction}

By calculating the Pearson Correlation with the enzyme percentages of all pathways for each pair of organisms, we transformed the percentage matrix into a distance matrix containing the metabolic distance between each pair of organisms. From this distance matrix, and using the PAUP* program version 4.0, we built a dendrogram using the neighbour-joining (NJ) algorithm. This dendrogram graphically represents the relationships between organisms based on their metabolism. We also built the dendrogram with the UPGMA algorithm, but this dendrogram was fairly similar to the one obtained by NJ.

\section{Bootstrap calculation}

To verify the dendrogram obtained, we developed a new method based on bootstrap calculations to check how robust each cluster was. From the primary percentage matrix, this method creates a certain number of distance matrices (a thousand in our case) by randomly selecting the metabolic pathways and allowing repetition. Using this group of matrices, we followed the same process as before and obtained a thousand trees. Using the consense program of the Phylip package, we calculated a consensus tree using the majority rule extended option with default parameters. The number of times each node is repeated indicates how reliable that cluster is.

\section{Discussion: \\ Dendrogram based on metabolism}

To ensure that the method developed was suitable for creating a dendrogram that would take into account at least the most basic taxonomic classification, we used it on 282 organisms (9 Eukaryota, 23 Archaea and 250 Bacteria) from the KEGG database. The evolution based on metabolic pathways is represented in the dendrogram in Figure 1. To make comparison easier, we have coloured the branches according to the taxonomic classification of their organism and classified the ISSN 0973-2063

Bioinformation 2(4): 135-144 (2007) open access

\section{Hypothesis}

organisms into fourteen groups. These groups, which differ in size, were defined by taking into account the clusters observed in Figure 1 and their bootstrap values. The result of the groupings and the taxonomic group to which each organism belongs are shown in Table 1 (supplementary material). In general, although this dendrogram does not follow the taxonomic classification perfectly, some large clusters encompass taxonomically related organisms while others appear as mixed clusters. Here we comment two causes that may lead to the grouping of mixed taxonomic clusters.

\section{Reduced genomes}

All Archaea are clustered together separately from the bacterial cluster, the only exception is Nanoarchaeum equitans Kin4-M (neq). Unlike the other Archaea we used to construct the dendrogram, this organism is an obligate symbiont. [17] It appears clustered with most of the intracellular or obligate parasites with a small genome found in our dendrogram (groups 4, 5 and 6). Parasitic organisms have reduced genomes, which means that their metabolic capacity has been lowered to a certain degree. This could explain the clustering of several parasite species even though they are phylogenetically distant. In a tree based on metabolic information, therefore, it should not be surprising to find that the only symbiont Archaea clusters with other parasites due to their particular metabolic characteristics.

\section{Metabolic similarity}

The firmicutes are grouped in two main groups, Lactobacillales (Group 9) and Bacillales (Group 10). Between these two groups there are smaller groups of other Firmicutes, one of which contains the Clostridia Thermoanaerobacter tengcongensis (tte) and Clostridium tetani (ctc) with two other organisms that do not belong to the Firmicutes phylum: Symbiobacterium thermophilum (sth) and Fusobacterium nucleatum (fnu). The location of F. nucleatum among Firmicutes can be explained by their shared metabolic pathways. [18] Despite being gram negative, $F$. nucleatum has been found to be more similar to gram positive bacteria than to gram negative ones. This is also true of $S$. thermophilum. The $16 \mathrm{~S}$ ribosomal DNA-based phylogeny suggested that this bacterium belongs to an unknown taxon in the gram-positive Actinobacteria [19], even though the traditional Gram-stain result indicates that it is gram negative. [20] Also, the proteins of $S$. thermophilum show a greater similarity to the proteins found in Firmicutes organisms, in particular to $T$. tengcongensis, than to those found in Actinobacteria. [20] The metabolic similarity between $S$. thermophilum and T. tengcongensis shown in figure 1 and the metabolic dissimilarity between $S$. thermophilum and the other Actinobacteria, combined with previous evidences [20, 21], suggest that $S$. thermophilum may be re-classified as Firmicutes, Clostridia. [21]

\section{Metabolic influence}

Not all kinds of metabolism influence our dendrogram in the same way. In Table 2 (supplementary material) we can see a distribution of the enzymes found in the defined groups in the different metabolic groups. For example, Carbohydrate Metabolism has much more influence on the dendrogram than Energy Metabolism, simply because it has many more enzymes and pathways. Also, some of these enzymes are not very useful for classifying organisms into the different clusters. A clear example is the enzymes that catalyse the reactions that produce the different Aminoacyl-tRNAs as they are present in nearly 


\section{Bioinformation}

\author{
www.bioinformation.net
}

by Biomedical Informatics Publishing Group

Hypothesis every group, even those with a reduced genome.

Table 2 (under supplementary material) also shows that for several groups some kinds of metabolisms stand out because of the high number of enzymes they possess compared to the main number of enzymes that the metabolic group has in all organisms. For example, Lipid metabolism in Metazoa (Group 13). This is explained by the presence of pathways such as the synthesis of Lecitin or Cholesterol. The contrary is also true. Some groups have fewer enzymes than most. Examples of this are the three parasitic groups (Group 4, 5 and 6). In their low enzyme values, we can clearly see the effects of genome evolutive reduction due to their parasitic nature.

\section{Limitations of metabolic-based methods}

By their nature, metabolic pathways databases are humandefined and may be quite inexact, especially when a metabolic pathway found in one species is generalized to another. Several alternative pathways that have not yet been discovered surely exist in different organisms. Therefore, when only one or a few enzymes from a metabolic pathway are missing in one species, an orthologous gene displacement needs to be considered before we can conclude that the pathway is incomplete. Moreover, when a new sequenced genome is annotated, a high percentage of its proteins are not mapped to any pathway. It may therefore be argued that metabolic databases, while extremely useful for reconstructing metabolic properties of organisms, cannot be used to reconstruct the tree of life. However, we have shown that, assuming that any metabolic prediction of a large group of organisms is still incomplete, the phylogenetic signal that it contains partially agrees with the taxonomic information of the species. A metabolic dendrogram of different species can therefore be used as an additional criterion that may help to correctly re-classify some species, as in the case of the Symbiobacterium thermophilum we described earlier.

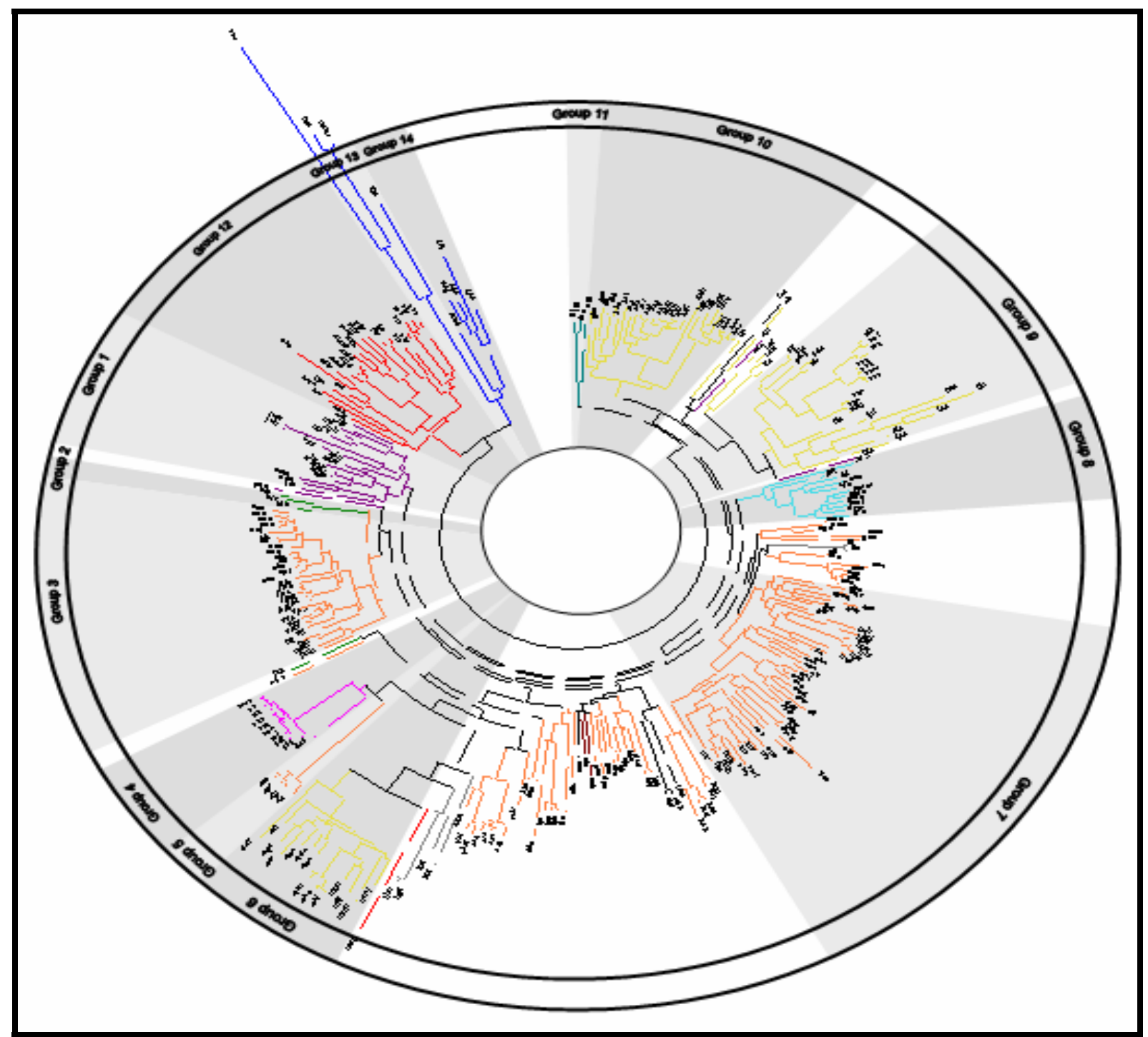

Figure 1: Dendrogram created from metabolic pathways by neighbour joining. The small squares represent nodes with more than 750 repetitions in the bootstrap analysis. The triangles are nodes with more than 900 repetitions. Taxonomic groups are marked by the same colouring: Actinobacteria in purple, Archaea in red, Bacteroidetes in green, Chlamydiae in pink, Cyanobacteria in pale blue, Deinococcus-Thermus in cyan, Eukaryota in dark blue, Firmicutes in yellow, Proteobacteria in orange, Spirochaeta in grey, and others in black. 


\section{Bioinformation}

\begin{abstract}
Conclusion:
We have developed a new method for constructing a dendrogram based on metabolic comparisons between species whose genome has been fully sequenced. Although the evolutionary signal that can be derived from metabolic data is not very strong, it is enough to obtain a rough sketch of the known taxonomic classification. We expect that the reconstruction of metabolic dendrograms may improve as more pathways are discovered and their enzymes are properly situated within those pathways. Until such a time metabolic-based dendrograms may be a useful addition when they are combined with other phylogenetic methods, allowing us to fine-tune dubious classifications that can not be accurately described by other methods.
\end{abstract}

\section{Acknowledgement:}

This work has been financed by the following project: "Ministerio de Ciencia y Tecnología” (Ref. BIO2003-07672). We thank Kevin Costello of the Language Service of the Rovira i Virgili University for his help with writing the manuscript.

\section{References:}

[01] C. R. Woese and G. E. Fox, Proc Natl Acad Sci., 74: 5088 (1997) [PMID: 270744]

[02] E. Pennisi, Science, 294: 634 (2001) [PMID: 11721026]

[03] S. Garcia-Vallve, et al., Nucleic Acids Res., 31: 187 (2003) [PMID: 12519978]

[04] S. Garcia-Vallve, et al., Genome Res., 10: 1719 (2000) [PMID: 12519978]

[05] W. F. Doolittle, Science, 284: 2124 (1999) [PMID: 10381871]

[06] J. P. Gogarten and J. P. Townsend, Nat Rev
Microbiol., 3: 679 (2005) [PMID: 16138096]

[07] E. Bapteste, et al., Trends Microbiol., 12: 406 (2004) [PMID: 15337161]

[08] B. E. Duthil, et al., Bioinformatics, 23: 815 (2007) [PMID: 17237036]

[09] F. Delsuc, et al., Nat Rev Genet., 6: 361 (2005) [PMID: 15861208]

[10] B. Snel, et al., Annu Rev Microbiol., 59: 191 (2005) [PMID: 16153168]

[11] F. D. Ciccarelli, et al., Science, 311: 1283 (2006) [PMID: 16513982]

[12] C. J. Creevey, et al., Proc $R$ Soc Lond B Biol Sci., 271: 2551 (2004) [PMID: 15615680]

[13] M. A. Huynen and P. Bork, Proc Natl Acad Sci., 95: 5849 (1998) [PMID: 9600883]

[14] S. Y. Shi, et al., Acta Biochim Biophys Sin., 37: 561 (2005) [PMID: 16077904]

[15] M. Kanehisa, et al., Nucleic Acids Res., 34: 354 (2006) [PMID: 16381885]

[16] R. Caspi, et al., Nucleic Acids Res., 34: 511 (2006) [PMID: 16381923]

[17] E. Waters, et al., Proc Natl Acad Sci., 100: 12984 (2003) [PMID: 14566062]

[18] V. Kapatral, et al., J Bacteriol., 184: 2005 (2002) [PMID: 11889109]

[19] M. Ohno, et al., Int. J. Syst. Evol. Microbio., 50: 1829 (2000) [PMID: 11034494]

[20] K. Ueda, et al., Nucleic Acids Res., 32: 4937 (2004) [PMID: 15383646]

[21] M. Wu, et al., PLoS Genet., 1: 65 (2005) [PMID: 16311624]

Edited by P. Kangueane

Citation: Marcet-Houben, et al., Bioinformation 2(4): 135-144 (2007) License statement: This is an open-access article, which permits unrestricted use, distribution, and reproduction in any medium, for non-commercial purposes, provided the original author and source are credited. 


\section{Bioinformation}

\section{Supplementary material}

\begin{tabular}{|c|c|c|c|c|c|}
\hline \multicolumn{3}{|c|}{ Eukaryota } & \multicolumn{3}{|c|}{ Firmicutes (cont) } \\
\hline Abbr & Organism Name & Group & Abbr & Organism Name & Group \\
\hline cal & Candida albicans SC5314 & 14 & spn & Streptococcus pneumoniae TIGR4 & 9 \\
\hline cme & Cyanidioschyzon merolae & 14 & spy & Streptococcus pyogenes M1 GAS & 9 \\
\hline ago & Eremothecium gossypii & 14 & spa & Streptococcus pyogenes MGAS10394 & 9 \\
\hline hsa & Homo sapiens & 13 & spg & Streptococcus pyogenes MGAS315 & 9 \\
\hline $\mathrm{mmu}$ & Mus musculus & 13 & spz & Streptococcus pyogenes MGAS5005 & 9 \\
\hline sce & Saccharomyces cerevisiae & 14 & spb & Streptococcus pyogenes MGAS6180 & 9 \\
\hline spo & Schizosaccharomyces pombe & 14 & spm & Streptococcus pyogenes MGAS8232 & 9 \\
\hline SSC & Sus scrofa & - & sps & Streptococcus pyogenes SSI-1 & 9 \\
\hline \multirow[t]{2}{*}{ xla } & Xenopus laevis & 13 & stc & Streptococcus thermophilus CNRZ1066 & 9 \\
\hline & Archaea: & & stl & Streptococcus thermophilus LMG 18311 & 9 \\
\hline Abbr & Organism Name & Group & tte & Thermoanaerobacter tengcongensis MB4 & - \\
\hline ape & Aeropyrum pernix & 12 & uur & Ureaplasma parvum serovar 3 str. ATCC 700970 & 6 \\
\hline afu & Archaeoglobus fulgidus DSM 4304 & 12 & & Proteobacteria: & \\
\hline hma & Haloarcula marismortui ATCC 43049 & 12 & Abbr & Organism Name & Group \\
\hline hal & Halobacterium sp. NRC-1 & 12 & aci & Acinetobacter sp. ADP1 & 7 \\
\hline mja & Methanocaldococcus jannaschii DSM 2661 & 12 & atc & Agrobacterium tumefaciens str. C58 (Cereon) & 7 \\
\hline mmp & Methanococcus maripaludis & 12 & atu & $\begin{array}{l}\text { Agrobacterium tumefaciens str. C58 } \\
\text { (U.Washington/Dupont) }\end{array}$ & 7 \\
\hline mka & Methanopyrus kandleri AV19 & 12 & ama & Anaplasma marginale str. St. Maries & - \\
\hline mac & Methanosarcina acetivorans C2A & 12 & eba & Azoarcus sp. EbN1 & 7 \\
\hline mba & Methanosarcina barkeri str. fusaro & 12 & bhe & Bartonella henselae str. Houston-1 & - \\
\hline mma & Methanosarcina mazei Go1 & 12 & bqu & Bartonella quintana str. Toulouse & - \\
\hline mth & $\begin{array}{c}\text { Methanothermobacter thermautotrophicus } \\
\text { str. Delta H }\end{array}$ & 12 & bba & Bdellovibrio bacteriovorus HD100 & - \\
\hline neq & Nanoarchaeum equitans Kin4-M & - & bbr & Bordetella bronchiseptica RB50 & 7 \\
\hline nph & Natronomonas pharaonis DSM 2160 & 12 & bpa & Bordetella parapertussis 12822 & 7 \\
\hline pto & Picrophilus torridus DSM 9790 & 12 & bpe & Bordetella pertussis Tohama I & 7 \\
\hline pai & Pyrobaculum aerophilum & 12 & bja & Bradyrhizobium japonicum USDA 110 & 7 \\
\hline pab & Pyrococcus abyssi GE5 & 12 & bmb & Brucella abortus biovar 1 str. 9-941 & 7 \\
\hline $\mathrm{pfu}$ & Pyrococcus furiosus DSM 3638 & 12 & bme & Brucella melitensis $16 M$ & 7 \\
\hline pho & Pyrococcus horikoshii OT3 & 12 & bmf & Brucella melitensis biovar Abortus 2308 & 7 \\
\hline sai & Sulfolobus acidocaldarius DSM 639 & 12 & bms & Brucella suis 1330 & 7 \\
\hline sso & Sulfolobus solfataricus P2 & 12 & bab & Buchnera aphidicola (Baizongia pistaciae) & - \\
\hline sto & Sulfolobus tokodaii str. 7 & 12 & buc & $\begin{array}{c}\text { Buchnera aphidicola str. APS (Acyrthosiphon } \\
\text { pisum) }\end{array}$ & - \\
\hline tko & Thermococcus kodakarensis KOD1 & 12 & bas & $\begin{array}{c}\text { Buchnera aphidicola str. Sg (Schizaphis } \\
\text { graminum) }\end{array}$ & - \\
\hline tac & Thermoplasma acidophilum DSM 1728 & 12 & bma & Burkholderia mallei ATCC 23344 & 7 \\
\hline \multirow[t]{2}{*}{ tvo } & Thermoplasma volcanium & 12 & bps & Burkholderia pseudomallei K96243 & 7 \\
\hline & Actinobacteria: & & bur & Burkholderia sp. 383 & 7 \\
\hline Abbr & Organism Name & Group & cjr & Campylobacter jejuni RM1221 & - \\
\hline blo & Bifidobacterium longum NCC2705 & - & cje & Campylobacter jejuni subsp. jejuni NCTC 11168 & - \\
\hline cdi & Corynebacterium diphtheriae NCTC 13129 & 1 & bfl & Candidatus Blochmannia floridanus & - \\
\hline cef & Corynebacterium efficiens YS-314 & 1 & bpn & $\begin{array}{l}\text { Candidatus Blochmannia pennsylvanicus str. } \\
\text { BPEN }\end{array}$ & - \\
\hline cgb & $\begin{array}{c}\text { Corynebacterium glutamicum ATCC } 13032 \\
\text { (Bielefeld) }\end{array}$ & 1 & pub & Candidatus Pelagibacter ubique HTCC1062 & 7 \\
\hline $\operatorname{cgl}$ & $\begin{array}{c}\text { Corynebacterium glutamicum ATCC } 13032 \\
\text { (Kyowa Hakko) }\end{array}$ & 1 & $\mathrm{ccr}$ & Caulobacter crescentus CB15 & 7 \\
\hline cjk & Corynebacterium jeikeium K411 & 1 & cvi & Chromobacterium violaceum ATCC 12472 & 7 \\
\hline $\operatorname{lxx}$ & Leifsonia xyli subsp. xyli str. СТСВ07 & 1 & cps & Colwellia psychrerythraea $34 \mathrm{H}$ & 7 \\
\hline
\end{tabular}




\section{Bioinformation}

\begin{tabular}{cc}
\hline mpa & Mycobacterium avium subsp. paratuberculosis \\
mbo & K-10 \\
mle & Mycobacterium bovis AF2122/97 \\
mtc & Mycobacterium tuberculosis CDC1551 \\
mtu & Mycobacterium tuberculosis H37Rv \\
nfa & Nocardia farcinica \\
pac & Propionibacterium acnes KPA171202 \\
sma & Streptomyces avermitilis MA-4680
\end{tabular}

SCO

sth

tfu

twh

tws

Abbr

bfs

bfr

bth

pgi

Abbr

$\mathrm{cmu}$

cta

ctr

cab

cса

сра

cpn

срj

cpt

pcu

Abbr

ava

gvi

ana

pmt

pmn

pma

$\mathrm{pmm}$

syc

syw

syn

tel

Abbr

dra

tth
Streptomyces coelicolor A3(2)

Symbiobacterium thermophilum IAM 14863

Thermobifida fusca $Y X$

Tropheryma whipplei str. Twist

Tropheryma whipplei TW08/27

Bacteroidetes:

Organism Name

Bacteroides fragilis NCTC 9343

Bacteroides fragilis $\mathrm{YCH} 46$

Bacteroides thetaiotaomicron VPI-5482

Porphyromonas gingivalis W83

Chlamydiae:

Organism Name

Chlamydia muridarum Nigg

Chlamydia trachomatis A/HAR-13

Chlamydia trachomatis D/UW-3/CX

Chlamydophila abortus S26/3

Chlamydophila caviae GPIC

Chlamydophila pneumoniae AR39

Chlamydophila pneumoniae CWL029

Chlamydophila pneumoniae J138

Chlamydophila pneumoniae TW-183

Parachlamydia sp. UWE25

Cyannobacteria:

Organism Name

Anabaena variabilis ATCC 29413

Gloeobacter violaceus

Nostoc sp. PCC 7120

Prochlorococcus marinus str. MIT 9313

Prochlorococcus marinus str. NATL2A

Prochlorococcus marinus subsp. marinus str. CCMP1375

Prochlorococcus marinus subsp. pastoris str. CCMP1986

Synechococcus elongatus PCC 6301

Synechococcus sp. WH 8102

Synechocystis sp. PCC 6803

Thermosynechococcus elongatus BP-1

\section{Deinococcus-Thermus:}

Organism Name

Deinococcus radiodurans $R 1$

Thermus thermophilus HB27 cbu

dar

dps

dvu

ecn

erg

eru

1 erw

1

-

1

1

1

Group $\mathrm{ftu}$

2 gsu

2 gox

2 hdu

- hit

hin

Group hhe

4 hpy

$4 \quad$ hpj

4 ilo

$4 \quad \operatorname{lpf}$

$4 \quad$ lpp

$4 \quad$ lpn

$4 \quad \mathrm{msu}$

4 mlo

4 mca

$4 \quad$ ngo

nme

Group nma

8 nwi

8 noc

8 neu

8 pmu

8 рса

8 ppr

8 plu

8 pha

8 pae

8 pfl

8 pfo

ppu

Group psp

11 psb

11 pst

140
Coxiella burnetii RSA 493

Dechloromonas aromatica RCB

Desulfotalea psychrophila LSv54

Desulfovibrio vulgaris subsp. vulgaris str. Hildenborough

Ehrlichia canis str. Jake

Ehrlichia ruminantium str. Gardel

Ehrlichia ruminantium str. Welgevonden (South Africa)

Ehrlichia ruminantium str. Welgevonden (France)

Erwinia carotovora subsp. atroseptica SCRI1043 3

Escherichia coli CFT073 3

Escherichia coli K12 W3110 3

Escherichia coli K12 MG1655 3

Escherichia coli O157:H7 3

Escherichia coli O157:H7 EDL933 3

Francisella tularensis subsp. tularensis

Geobacter sulfurreducens PCA

Gluconobacter oxydans $621 \mathrm{H}$

Haemophilus ducreyi $35000 \mathrm{HP}$

Haemophilus influenzae 86-028NP

Haemophilus influenzae Rd KW20

Helicobacter hepaticus ATCC 51449

Helicobacter pylori 26695

Helicobacter pylori $J 99$

Idiomarina loihiensis L2TR

Legionella pneumophila str. Lens

Legionella pneumophila str. Paris

Legionella pneumophila subsp. pneumophila str. Philadelphia 1

Mannheimia succiniciproducens MBEL55E 3

Mesorhizobium loti MAFF303099 7

Methylococcus capsulatus str. Bath $\quad 7$

Neisseria gonorrhoeae FA 1090

Neisseria meningitidis MC58 -

Neisseria meningitidis Z2491 -

Nitrobacter winogradskyi $\mathrm{Nb}-255 \quad 7$

Nitrosococcus oceani ATCC $19707 \quad 7$

Nitrosomonas europaea ATCC $19718 \quad 7$

Pasteurella multocida subsp. multocida str. $\quad 3$ Pm70

Pelobacter carbinolicus DSM 2380

Photobacterium profundum 3

Photorhabdus luminescens subsp. laumondii $\quad 3$ TTO1

$\begin{array}{ll}\text { Pseudoalteromonas haloplanktis TAC125 } & 7\end{array}$

Pseudomonas aeruginosa PAO1 7

Pseudomonas fluorescens Pf-5 7

Pseudomonas fluorescens PfO-1 7

Pseudomonas putida KT2440 7

Pseudomonas syringae pv. phaseolicola 1448A $\quad 7$

\begin{tabular}{ll} 
Pseudomonas syringae pv. syringae B728a $\quad 7$ \\
\hline
\end{tabular}

Pseudomonas syringae pv. tomato str. DC3000 7 


\section{Bioinformation}

\begin{tabular}{|c|c|c|c|c|c|}
\hline $\mathrm{ttj}$ & Thermus thermophilus HB8 & 11 & par & Psychrobacter arcticus 273-4 & 7 \\
\hline & Firmicutes: & & reu & Ralstonia eutropha JMP134 & 7 \\
\hline Abbr & Organism Name & Group & rso & Ralstonia solanacearum GMI1000 & 7 \\
\hline baa & Bacillus anthracis str. A2012 & 10 & rsp & Rhodobacter sphaeroides 2.4.1 & 7 \\
\hline ban & Bacillus anthracis str. Ames & 10 & rpa & Rhodopseudomonas palustris CGA009 & 7 \\
\hline bar & Bacillus anthracis str. 'Ames Ancestor' & 10 & rco & Rickettsia conorii str. Malish 7 & 5 \\
\hline bat & Bacillus anthracis str. Sterne & 10 & rfe & Rickettsia felis URRWXCal2 & 5 \\
\hline bca & Bacillus cereus ATCC 10987 & 10 & rpr & Rickettsia prowazekii str. Madrid E & 5 \\
\hline bce & Bacillus cereus ATCC 14579 & 10 & rty & Rickettsia typhi str. Wilmington & 5 \\
\hline bcz & Bacillus cereus E33L & 10 & $\mathrm{sec}$ & $\begin{array}{l}\text { Salmonella enterica subsp. enterica serovar } \\
\text { Choleraesuis str. SC-B67 }\end{array}$ & 3 \\
\hline bcl & Bacillus clausii KSM-K16 & 10 & spt & $\begin{array}{c}\text { Salmonella enterica subsp. enterica serovar } \\
\text { Paratyphi A str. ATCC } 9150\end{array}$ & 3 \\
\hline bha & Bacillus halodurans & 10 & sty & $\begin{array}{c}\text { Salmonella enterica subsp. enterica serovar } \\
\text { Typhi str. CT18 }\end{array}$ & 3 \\
\hline bld & Bacillus licheniformis DSM13 & 10 & stt & $\begin{array}{c}\text { Salmonella enterica subsp. enterica serovar } \\
\text { Typhi Ty2 }\end{array}$ & 3 \\
\hline bli & Bacillus licheniformis ATCC 14580 & 10 & stm & Salmonella typhimurium LT2 & 3 \\
\hline bsu & Bacillus subtilis subsp. subtilis str. 168 & 10 & son & Shewanella oneidensis MR-1 & 7 \\
\hline btk & $\begin{array}{c}\text { Bacillus thuringiensis serovar konkukian str. } \\
97-27\end{array}$ & 10 & sfx & Shigella flexneri $2 a$ str. $2457 T$ & 3 \\
\hline $\mathrm{cac}$ & Clostridium acetobutylicum ATCC 824 & - & sfl & Shigella flexneri $2 a$ str. 301 & 3 \\
\hline сре & Clostridium perfringens str. 13 & 9 & $\operatorname{ssn}$ & Shigella sonnei Ss046 & 3 \\
\hline ctc & Clostridium tetani E88 & - & sil & Silicibacter pomeroyi DSS-3 & 7 \\
\hline efa & Enterococcus faecalis V583 & 9 & sme & Sinorhizobium meliloti 1021 & 7 \\
\hline gka & Geobacillus kaustophilus HTA426 & 10 & tbd & Thiobacillus denitrificans ATCC 25259 & 7 \\
\hline lac & Lactobacillus acidophilus NCFM & 9 & vch & Vibrio cholerae O1 biovar eltor str. N16961 & 3 \\
\hline ljo & Lactobacillus johnsonii NCC 533 & 9 & vfi & Vibrio fischeri ES114 & 3 \\
\hline lpl & Lactobacillus plantarum WCFS1 & 9 & vpa & Vibrio parahaemolyticus RIMD 2210633 & 3 \\
\hline lsa & Lactobacillus sakei subsp. sakei $23 K$ & 9 & vvu & Vibrio vulnificus CMCP6 & 3 \\
\hline lla & Lactococcus lactis subsp. lactis Il1403 & 9 & vvy & Vibrio vulnificus YJ016 & 3 \\
\hline $\operatorname{lin}$ & Listeria innocua Clip11262 & - & wbr & $\begin{array}{l}\text { Wigglesworthia glossinidia endosymbiont of } \\
\text { Glossina brevipalpis }\end{array}$ & - \\
\hline $\operatorname{lmo}$ & Listeria monocytogenes EGD-e & - & wol & $\begin{array}{c}\text { Wolbachia endosymbiont of Drosophila } \\
\text { melanogaster }\end{array}$ & - \\
\hline $\operatorname{lmf}$ & Listeria monocytogenes str. $4 b$ F2365 & - & wbm & $\begin{array}{c}\text { Wolbachia endosymbiont strain TRS of Brugia } \\
\text { malayi }\end{array}$ & - \\
\hline $\mathrm{mfl}$ & Mesoplasma florum L1 & 6 & wsu & Wolinella succinogenes DSM 1740 & - \\
\hline mga & Mycoplasma gallisepticum $R$ & 6 & хас & Xanthomonas axonopodis pv. citri str. 306 & - \\
\hline mge & Mycoplasma genitalium G37 & 6 & xcb & Xanthomonas campestris pv. campestris str. 8004 & - \\
\hline mhy & Mycoplasma hyopneumoniae 232 & 6 & XCC & $\begin{array}{c}\text { Xanthomonas campestris pv. campestris str. } \\
\text { ATCC } 33913\end{array}$ & - \\
\hline mhp & Mycoplasma hyopneumoniae 7448 & 6 & $\mathrm{xcV}$ & $\begin{array}{c}\text { Xanthomonas campestris pv. vesicatoria str. 85- } \\
10\end{array}$ & - \\
\hline mhj & Mycoplasma hyopneumoniae $J$ & 6 & xoo & Xanthomonas oryzae pv. oryzae KACC10331 & - \\
\hline mmo & Mycoplasma mobile $163 \mathrm{~K}$ & 6 & $\mathrm{xfa}$ & Xylella fastidiosa $9 a 5 c$ & - \\
\hline mmy & $\begin{array}{l}\text { Mycoplasma mycoides subsp. mycoides SC str. } \\
\text { PG1 }\end{array}$ & 6 & $\mathrm{xft}$ & Xylella fastidiosa Temecula1 & - \\
\hline mpe & Mycoplasma penetrans & 6 & ypm & Yersinia pestis biovar Medievalis str. 91001 & 3 \\
\hline mpn & Mycoplasma pneumoniae M129 & 6 & ype & Yersinia pestis CO92 & 3 \\
\hline mpu & Mycoplasma pulmonis UAB CTIP & 6 & ypk & Yersinia pestis KIM & 3 \\
\hline msy & Mycoplasma synoviae 53 & 6 & yps & Yersinia pseudotuberculosis IP 32953 & 3 \\
\hline oih & Oceanobacillus iheyensis HTE831 & 10 & zmo & Zymomonas mobilis subsp. mobilis ZM4 & - \\
\hline poy & Onion yellows phytoplasma & 6 & & Spirochaetas: & \\
\hline sab & Staphylococcus aureus RF122 & 10 & Abbr & Organism Name & Group \\
\hline sac & Staphylococcus aureus subsp. aureus COL & 10 & lic & $\begin{array}{l}\text { Leptospira interrogans serovar Copenhageni str. } \\
\text { Fiocruz L1-130 }\end{array}$ & - \\
\hline
\end{tabular}




\section{Bioinformation}

\begin{tabular}{|c|c|c|c|c|c|}
\hline sar & $\begin{array}{l}\text { Staphylococcus aureus subsp. aureus } \\
\text { MRSA252 }\end{array}$ & 10 & lil & Leptospira interrogans serovar Lai str. 56601 & - \\
\hline sas & $\begin{array}{c}\text { Staphylococcus aureus subsp. aureus } \\
\text { MSSA476 }\end{array}$ & 10 & bbu & Borrelia burgdorferi B31 & - \\
\hline sav & Staphylococcus aureus subsp. aureus Mu50 & 10 & bga & Borrelia garinii $\mathrm{PBi}$ & - \\
\hline sam & Staphylococcus aureus subsp. aureus MW2 & 10 & tde & Treponema denticola ATCC 35405 & - \\
\hline sau & Staphylococcus aureus subsp. aureus N315 & 10 & tpa & Treponema pallidum subsp. pallidum str. Nichols & - \\
\hline sep & Staphylococcus epidermidis ATCC 12228 & 10 & & Others: & \\
\hline ser & Staphylococcus epidermidis RP62A & 10 & Abbr & Organism Name & Group \\
\hline sha & Staphylococcus haemolyticus JCSC1435 & 10 & aae & Aquifex aeolicus VF5 & - \\
\hline ssp & $\begin{array}{l}\text { Staphylococcus saprophyticus subsp. } \\
\text { saprophyticus }\end{array}$ & 10 & cch & Chlorobium chlorochromatii CaD3 & - \\
\hline sag & Streptococcus agalactiae 2603V/R & 9 & cte & Chlorobium tepidum TLS & - \\
\hline sak & Streptococcus agalactiae A909 & 9 & det & Dehalococcoides ethenogenes 195 & - \\
\hline san & Streptococcus agalactiae NEM316 & 9 & deh & Dehalococcoides sp. CBDB1 & - \\
\hline smu & Streptococcus mutans UA159 & 9 & fnu & $\begin{array}{l}\text { Fusobacterium nucleatum subsp. nucleatum } \\
\text { ATCC } 25586\end{array}$ & - \\
\hline spr & Streptococcus pneumoniae R6 & 9 & tma & Thermotoga maritima MSB8 & - \\
\hline
\end{tabular}

Table 1: Abbreviation and taxonomic classification of the 282 organisms included in the analysis 


\section{Hypothesis}

\begin{tabular}{|c|c|c|c|c|c|c|c|c|c|c|c|c|c|c|c|c|c|c|c|c|c|c|c|c|c|c|}
\hline & $\begin{array}{c}\text { Taxonomic } \\
\text { classification } \\
\text { (organisms } \\
\text { belonging to } \\
\text { classification/ } \\
\text { total organisms } \\
\text { group) }\end{array}$ & & 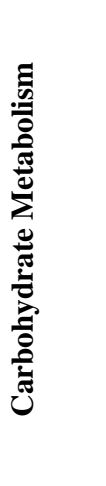 & & 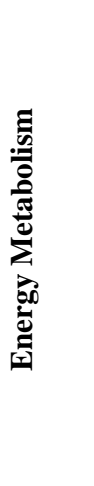 & & & & 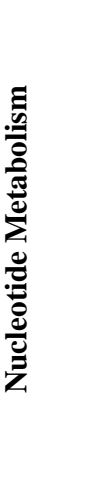 & & 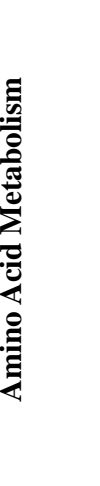 & & 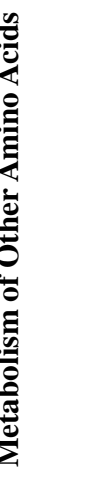 & (1) & 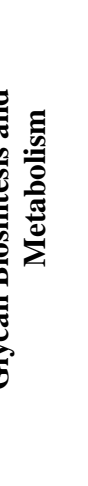 & & 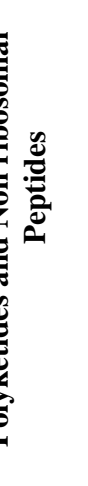 & & 告 & 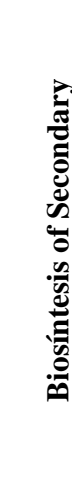 & 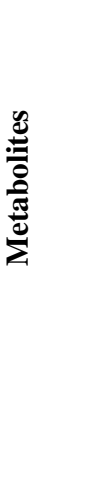 & & 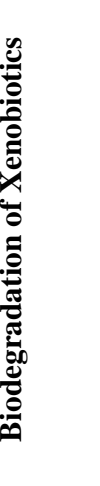 & & 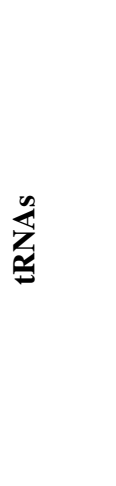 & 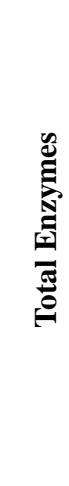 \\
\hline Group1 & $\begin{array}{c}\text { Actinobacteria } \\
(17 / 17)\end{array}$ & 176 & $(25.9)$ & 28 & $(4.1)$ & 22 & $(3.2)$ & 51 & (7.5) & 207 & (30.5) & 20 & (2.9) & 8 & $(1.2)$ & 4 & $(0.5)$ & 116 & $(17.0)$ & 15 & (2.3) & 14 & $(2.0)$ & 20 & (2.9) & 679 \\
\hline Group2 & $\begin{array}{c}\text { Bacteroidetes } \\
(3 / 3)\end{array}$ & 201 & $(29.2)$ & 34 & (4.9) & 12 & (1.8) & 48 & $(7.0)$ & 199 & (28.9) & 18 & (2.7) & 23 & (3.4) & 4 & $(0.6)$ & 113 & (16.4) & 13 & (1.9) & 0 & $(0.0)$ & 22 & $(3.2)$ & 688 \\
\hline Group3 & $\begin{array}{l}\text { Enterobacteria, } \\
\text { Vibrionales, } \\
\text { Pasteurellales } \\
(29 / 29)\end{array}$ & 216 & $(27.8)$ & 38 & (4.9) & 21 & (2.8) & 52 & (6.6) & 224 & (28.8) & 27 & (3.4) & 25 & (3.2) & 2 & $(0.3)$ & 129 & (16.6) & 14 & (1.8) & 7 & $(0.9)$ & 22 & $(2.8)$ & 777 \\
\hline Group4 & $\begin{array}{l}\text { Chlamidiae } \\
\text { (10/10) }\end{array}$ & 117 & $(33.2)$ & 11 & (3.1) & 9 & $(2.6)$ & 20 & (5.6) & 82 & (23.3) & 5 & (1.6) & 19 & (5.4) & 0 & $(0.1)$ & 63 & (17.8) & 6 & (1.7) & 0 & $(0.0)$ & 20 & (5.7) & 352 \\
\hline Group5 & $\begin{array}{l}\text { Rickettsia } \\
\text { (4/4) }\end{array}$ & 71 & (28.1) & $8 \mid$ & (3.4) & 6 & (2.6) & 20 & $(8.0)$ & 53 & (21.0) & 7 & $(2.8)$ & 20 & $(8.0)$ & 1 & $(0.4)$ & 38 & (15.3) & 5 & (1.9) & 1 & $(0.6)$ & 20 & $(8.0)$ & 251 \\
\hline Group6 & $\begin{array}{l}\text { Mollicutes } \\
\text { (14/14) }\end{array}$ & 96 & (50.9) & 10 & (5.3) & 4 & (1.9) & 14 & $(7.2)$ & 19 & $(10.0)$ & 4 & (2.1) & 0 & $(0.0)$ & 0 & $(0.0)$ & 21 & (10.9) & 1 & $(0.6)$ & 1 & $(0.3)$ & 21 & (10.9) & 189 \\
\hline Group7 & $\begin{array}{c}\text { Proteobacteria } \\
\text { (43/43) }\end{array}$ & 188 & $(24.3)$ & 36 & $(4.7)$ & 27 & (3.5) & 52 & (6.8) & 240 & (31.1) & 25 & (3.3) & 21 & (2.8) & 3 & $(0.4)$ & 120 & $(15.6)$ & 16 & $(2.0)$ & 21 & $(2.7)$ & 21 & $(2.7)$ & 771 \\
\hline Group8 & $\begin{array}{c}\text { Cyanobacteria } \\
(11 / 11)\end{array}$ & 160 & $(24.7)$ & 29 & $(4.4)$ & 17 & $(2.6)$ & 48 & $(7.4)$ & 190 & $(29.4)$ & 18 & $(2.8)$ & 15 & $(2.3)$ & 4 & $(0.5)$ & 123 & $(19.0)$ & 19 & $(3.0)$ & 4 & $(0.6)$ & 20 & $(3.2)$ & 647 \\
\hline Group9 & $\begin{array}{c}\text { Lactobacillales } \\
(21 / 22)\end{array}$ & 152 & (31.6) & 25 & $(5.2)$ & 15 & (3.1) & 47 & (9.8) & 126 & (26.3) & 14 & (2.9) & 7 & (1.5) & 3 & $(0.7)$ & 53 & (11.1) & 13 & (2.8) & 2 & $(0.5)$ & 21 & $(4.4)$ & 479 \\
\hline Group10 & $\begin{array}{c}\text { Bacillales } \\
(26 / 26)\end{array}$ & 193 & (26.9) & 32 & $(4.4)$ & 27 & (3.7) & 50 & $(7.0)$ & 229 & (31.9) & 27 & (3.7) & 7 & $(1.0)$ & 2 & $(0.3)$ & 108 & $(15.0)$ & 16 & $(2.2)$ & 7 & $(1.0)$ & 21 & $(2.9)$ & 718 \\
\hline Group11 & $\begin{array}{c}\text { Deinococcus- } \\
\text { Thermus } \\
(3 / 3)\end{array}$ & 175 & (25.7) & 34 & $(5.0)$ & 23 & (3.3) & 50 & (7.3) & 227 & $(33.2)$ & 18 & $(2.6)$ & 7 & $(1.0)$ & 2 & $(0.3)$ & 102 & $(15.0)$ & 13 & $(2.0)$ & 9 & (1.4) & 22 & $(3.2)$ & 682 \\
\hline $\begin{array}{l}\text { ISS } \\
\text { Bio }\end{array}$ & $\begin{array}{l}0973-2063 \\
\text { formation 2(4): 135- }\end{array}$ & $4(2$ & & & & & & & & & & 143 & & & & & & & & & & & & & & \\
\hline
\end{tabular}




\section{Bioinformation}

www.bioinformation.net

open access

\section{Hypothesis}

\begin{tabular}{|c|c|c|c|c|c|c|c|c|c|c|c|c|c|c|c|c|c|c|c|c|c|c|c|c|c|c|}
\hline Group12 & $\begin{array}{c}\text { Archaea } \\
(23 / 23)\end{array}$ & 122 & $(25.2)$ & 19 & $(4.0)$ & 11 & $(2.4)$ & 44 & $(9.0)$ & 168 & (34.9) & 11 & (2.2) & 2 & $(0.5)$ & 3 & $(0.6)$ & 64 & (13.2) & 13 & $(2.7)$ & 6 & (1.3) & 19 & $(4.0)$ & 483 \\
\hline Group13 & $\begin{array}{c}\text { Metazoa } \\
(3 / 3)^{*}\end{array}$ & 202 & (28.8) & 22 & $(3.1)$ & 71 & (10.1) & 52 & (7.4) & 194 & $(27.7)$ & 20 & (2.9) & 14 & $(2.0)$ & 1 & $(0.1)$ & 77 & (11.1) & 17 & $(2.5)$ & 8 & $(1.1)$ & 21 & (3.0) & 699 \\
\hline Group14 & $\begin{array}{l}\text { Fungi } \\
(4 / 5)\end{array}$ & 183 & (25.9) & 29 & $(4.1)$ & 31 & $(4.4)$ & 49 & $(7.0)$ & 237 & (33.6) & 21 & (3.0) & 14 & $(2.0)$ & 1 & $(0.1)$ & 93 & (13.2) & 16 & (2.3) & 9 & $(1.2)$ & 22 & (3.1) & 706 \\
\hline
\end{tabular}

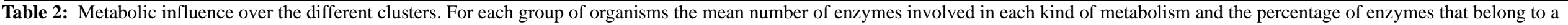

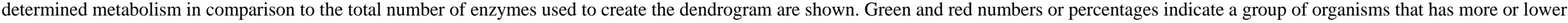

enzymes of a kind of metabolism than most of the other groups. * Sus scrofa (ssc) was excluded from these data because of the lack of KEGG numbers on important metabolic protein 